\title{
Contribution of an SFK-Mediated Signaling Pathway in the Dorsal Hippocampus to Cocaine-Memory Reconsolidation in Rats
}

\author{
Audrey M Wells', Xiaohu Xie ${ }^{2}$, Jessica A Higginbotham ${ }^{3}$, Amy A Arguello ${ }^{3}$, Kati L Healey ${ }^{4}$, Megan Blanton ${ }^{4}$ \\ and Rita A Fuchs*,3 \\ 'Behavioral Genetics Laboratory, Department of Psychiatry, Harvard Medical School, McLean Hospital, Belmont, CA, USA; ${ }^{2}$ Department of \\ Chemistry, North Carolina State University, Raleigh, NC, USA; ${ }^{3}$ Department of Integrative Physiology and Neuroscience, Washington State \\ University College of Veterinary Medicine, Pullman, WA, USA; ${ }^{4}$ Department of Psychology, University of North Carolina at Chapel Hill, Chapel Hill, \\ NC, USA
}

\begin{abstract}
Environmentally induced relapse to cocaine seeking requires the retrieval of context-response-cocaine associative memories. These memories become labile when retrieved and must undergo reconsolidation into long-term memory storage to be maintained. Identification of the molecular underpinnings of cocaine-memory reconsolidation will likely facilitate the development of treatments that mitigate the impact of cocaine memories on relapse vulnerability. Here, we used the rat extinction-reinstatement procedure to test the hypothesis that the Src family of tyrosine kinases (SFK) in the dorsal hippocampus (DH) critically controls contextual cocaine-memory reconsolidation. To this end, we evaluated the effects of bilateral intra-DH microinfusions of the SFK inhibitor, PP2 (62.5 ng per $0.5 \mu \mathrm{l}$ per hemisphere), following re-exposure to a cocaine-associated (cocaine-memory reactivation) or an unpaired context (no memory reactivation) on subsequent drug context-induced instrumental cocaine-seeking behavior. We also assessed alterations in the phosphorylation state of SFK targets, including GluN2A and GluN2B N-methyl-D-aspartate (NMDA) and GluA2 $\alpha$-amino-3-hydroxy-5-methyl-4-isoxazolepropionic acid receptor subunits at the putative time of memory restabilization and following PP2 treatment. Finally, we evaluated the effects of intraDH PEAQX (2.5 $\mu$ g per $0.5 \mu$ per hemisphere), a GluN2A-subunit-selective NMDAR antagonist, following, or in the absence of, cocainememory reactivation on subsequent drug context-induced cocaine-seeking behavior. GluN2A phosphorylation increased in the DH during putative memory restabilization, and intra-DH PP2 treatment inhibited this effect. Furthermore, PP2 - as well as PEAQX-attenuated subsequent drug context-induced cocaine-seeking behavior, in a memory reactivation-dependent manner, relative to $V E H$. These findings suggest that hippocampal SFKs contribute to the long-term stability of cocaine-related memories that underlie contextual stimulus control over cocaine-seeking behavior.
\end{abstract}

Neuropsychopharmacology (2016) 4I, 675-685; doi: I0.1038/npp.20 I5.217; published online I2 August 2015

\section{INTRODUCTION}

Cocaine-associated environments evoke craving and promote relapse, thereby impeding the successful treatment of cocaine addiction (Gawin and Kleber, 1986; Childress et al, 1988). Implicit in this is the retrieval and utilization of context-response-cocaine memories (Sorg, 2012; Tronson and Taylor, 2013). It is theorized that associative memories are restabilized into long-term memory (LTM) storage via a protein synthesis-dependent memory reconsolidation process following their retrieval and destabilization (Nader et al, 2000a). Therefore, it is possible that destabilized

\footnotetext{
*Correspondence: Dr RA Fuchs, Department of Integrative Physiology and Neuroscience, Washington State University College of Veterinary Medicine, PO Box 647620, Pullman, WA 99164-7620, USA,

Tel: + I 509335 6I64, Fax: + I 509335 4650,

E-mail: ritafuchs@vetmed.wsu.edu

Received 28 January 20 I5; revised I 3 July 20 I 5; accepted I 5 July 20 I5; accepted article preview online 23 July 2015
}

cocaine-related memories can be therapeutically modified by interfering with memory reconsolidation (Tronson and Taylor, 2013).

The dorsal hippocampus (DH) regulates the reconsolidation of contextual cocaine memories that direct instrumental cocaine-seeking behavior (Ramirez et al, 2009) through interactions with the basolateral amygdala (BLA; Wells et al, 2011). However, unlike the BLA, the DH contributes to cocaine-memory reconsolidation in a protein synthesisindependent manner that is poorly understood (Ramirez et al, 2009). Characterizing the intracellular signaling pathways in the DH that control cocaine-memory reconsolidation fills a gap in our understanding and may advance the development of novel therapeutics for relapse prevention.

$\mathrm{N}$-methyl-D-aspartate receptor (NMDAR) stimulation is necessary for drug-memory reconsolidation in several experimental procedures (Kelley et al, 2007; Sadler et al, 2007; Brown et al, 2008; Milton et al, 2008a). NMDAR function is regulated by the Src family of tyrosine kinases 
(SFKs; Salter and Kalia, 2004), which includes Src, Fyn, Lyn, Yes, and Lck (Ohinishi et al, 2011). SFKs phosphorylate GluN2A (Nakazawa et al, 2001; Taniguchi et al, 2009) and GluN2B NMDAR subunits (Nakazawa et al, 2001; Prybylowski et al, 2005; Stramiello and Wagner, 2008) to promote synaptic plasticity and memory (Purcell and Carew, 2003; Ohinishi et al, 2011). Specifically, SFK-mediated phosphorylation of GluN2A subunits is associated with synaptic strengthening and long-term potentiation (LTP; Yang et al, 2012). Additionally, SFK activation in the dorsal striatum and $\mathrm{DH}$ is necessary for drug-induced behavioral phenomena, such as drug-primed ethanol-seeking (Wang et al, 2010) and context-elicited cocaine-seeking behaviors (Xie et al, 2013), respectively. However, the role of SFKs in drug-memory reconsolidation is unknown.

The present study tested the hypothesis that SFK-mediated signaling in the DH is necessary for the reconsolidation of contextual cocaine memories that promote instrumental cocaine-seeking behavior. In Experiment 1, we evaluated (a) the effects of SFK inhibition in the DH during putative memory reconsolidation on subsequent drug context-induced cocaine-seeking behavior and (b) whether these effects depended on explicit memory reactivation. In Experiment 2, we used quantitative western blotting to determine whether SFK inhibition in the DH during cocaine-memory reconsolidation attenuates GluN2A NMDAR subunit phosphorylation. We also evaluated changes in alternate targets of SFKs: GluN2B NMDA and GluA2 AMPA ( $\alpha$-amino-3-hydroxy-5-methyl-4isoxazolepropionic acid) receptor subunits (Hayashi et al, 1999; Hayashi and Huganir, 2004). Finally, in Experiment 3, we assessed the effects of a GluN2A-preferring NMDAR antagonist in the $\mathrm{DH}$ on cocaine-memory reconsolidation.

\section{MATERIALS AND METHODS}

\section{Animals}

Male Sprague-Dawley rats $(N=112 ; 275-300$ g; Charles River Laboratories, Wilmington, MA or Simonsen Laboratories, Gilroy, CA) were individually housed in a temperature- and humidity-controlled vivarium under a reversed light-dark cycle. Rats were fed $20-25 \mathrm{~g}$ of rat chow daily. Water was available ad libitum. The housing and treatment of rats followed the Guide for the Care and Use of Laboratory Animals (National Research Council, 2011) and were approved by the IACUC of the University of North Carolina Chapel Hill and Washington State University.

\section{Food Training and Surgery}

To expedite cocaine self-administration, rats were first trained to lever press for $45 \mathrm{mg}$ food pellets (Noyes, Lancaster, $\mathrm{NH}$ ) during an overnight session in sound-attenuated operant conditioning chambers (Coulbourn Instruments, Allentown, PA). Forty-eight hours later, rats were surgically implanted with intravenous jugular catheters and with 26-gauge stainless-steel guide cannulae (Plastics One, Roanoke, VA) aimed bilaterally at the $\mathrm{DH}$ (angled by $15^{\circ}$; $\mathrm{AP}-3.4, \mathrm{ML} \pm 3.1$, and DV $-2.15 \mathrm{~mm}$ ) or the adjacent trunk region of the somatosensory cortex (SStr; angled by $15^{\circ}$; AP $-3.4, \mathrm{ML} \pm 3.1$, and DV $-0.65 \mathrm{~mm}$ ). Surgical and postoperative procedures are further described in Supplementary Methods.

\section{Self-Administration and Extinction Training}

Rats were randomly assigned to one of two distinctly different contexts (Context A or B, see Supplementary Table S1) for self-administration training, which took place during daily, $2 \mathrm{~h}$ sessions during the dark cycle. During these sessions, responses on a designated active lever produced cocaine infusions (cocaine hydrochloride; $0.15 \mathrm{mg}$ per $0.05 \mathrm{ml}$ per infusion, intravenously; NIDA, Research Triangle Park, NC) under an FR1 - 20 s time-out reinforcement schedule, as described previously (Fuchs et al, 2009). Presses on a second, inactive lever were recorded but had no scheduled consequences. Training continued until rats met the acquisition criterion (ie, $\geqslant 10$ sessions with $\geqslant 10$ cocaine infusions per session).

Next, rats received seven daily $2 \mathrm{~h}$ extinction-training sessions in the other context. Lever presses were recorded but had no programmed consequences. Rats in Experiments 1 and 3 were adapted to the intracranial microinfusion procedure after session 4. Rats in Experiment 2 were adapted to the same procedure after sessions 6 and 7 to minimize the potential effects of infusion stress on protein expression (see Supplementary Methods).

\section{Experiment 1a}

In Experiment $1 \mathrm{a}$, we evaluated whether intra-DH microinfusions of 4-Amino-3-(4-chlorophenyl)-1-(t-butyl)1H-pyrazolo[3,4-d]pyrimidine, 4-Amino-5-(4-chlorophenyl)-7-(t-butyl)pyrazolo[3,4-d]pyrimidine (PP2), an ATPcompetitive inhibitor of SFKs (Hanke et al, 1996), administered during the putative time of cocaine-memory reconsolidation would attenuate subsequent drug context-induced cocaine-seeking behavior. One day after the seventh extinction-training session, rats were re-exposed to the previously cocaine-paired context for $15 \mathrm{~min}$ (ie, cocainememory reactivation; see experimental timeline in Figure 1a) to trigger the destabilization and reconsolidation of contextresponse-cocaine memories (Fuchs et al, 2009). The number of extinction training sessions was uniform to control for memory age, a strong predictor of a memory's sensitivity to destabilization (Suzuki et al, 2004; Inda et al, 2011), at the time of memory reactivation. Lever pressing was recorded but was not reinforced during the memory reactivation session to prevent possible acute cocaine-induced potentiation of SFK-mediated GluN2A subunit phosphorylation (as reported in the ventral tegmental area; Schumann et al, 2009) that is independent of memory reactivation. Immediately after this session, rats received bilateral microinfusions of $0.1 \%$ DMSO VEH or PP2 (62.5 ng per $0.5 \mu \mathrm{l}$ per hemisphere) into the DH (see Supplementary Methods). This intra-DH PP2 dose was selected because it attenuates the expression of drug context-induced cocaine-seeking behavior (Xie et al, 2013). Treatment assignment was counterbalanced based on cocaine history.

Beginning the following day, rats received additional daily $2 \mathrm{~h}$ extinction sessions until they reached the extinction criterion (ie, $\leqslant 25$ active lever responses per session on $\geqslant 2$ consecutive days). Assessment of lever pressing in the extinction context can reveal the possible nonspecific effects of manipulations on general activity and memory (eg, context discrimination impairment because of general 


\begin{tabular}{|c|c|c|c|c|}
\hline $12345678910^{\circ} 1234567$ & $\begin{array}{c}\text { 15-min re-exposure } \\
\text { to COC-paired ctx }\end{array}$ & $12^{\#}$ & $\begin{array}{c}\text { 1-h test } \\
\text { of cocaine seeking }\end{array}$ \\
PP2 or VEH \\
into the DH
\end{tabular}

b
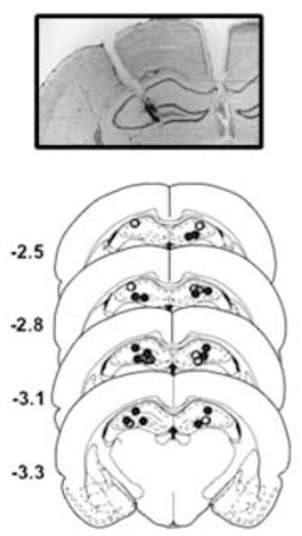

C
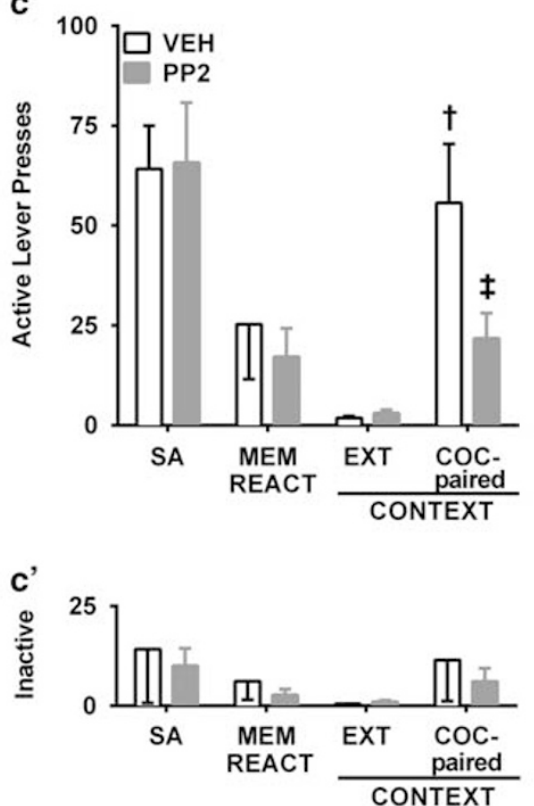

d

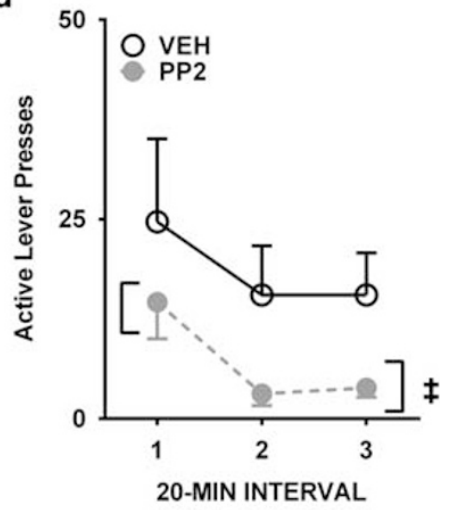

d'

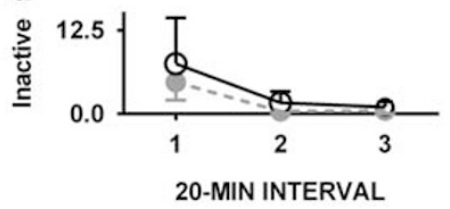

Figure I Inhibition of Src family of tyrosine kinases (SFKs) in the dorsal hippocampus (DH) following cocaine-memory reactivation attenuates subsequent drug context-induced cocaine seeking. (a) Schematic depicting the timeline for Experiment la. Cocaine self-administration (SA) sessions took place in a distinct environmental context until rats reached the acquisition criterion (* $\geqslant 10$ infusions per session during $\geqslant 10$ sessions). Extinction training occurred in a different environmental context. Next, rats were re-exposed to the cocaine-paired (COC-paired) context (cocaine-memory reactivation) for 15 min to trigger cocaine memory destabilization and subsequent reconsolidation. Immediately after the session, rats received bilateral microinfusions of PP2 (a nonselective SFK inhibitor, $62.5 \mathrm{ng}$ per $0.5 \mu \mathrm{l}$ per hemisphere) or $0.1 \%$ dimethyl sulfoxide (DMSO) vehicle (VEH; $0.5 \mu$ l per hemisphere) into the DH. Next, the groups received additional extinction training until they met the extinction criterion ( ${ }^{\#} \leqslant 25$ non-reinforced active lever responses per session during $\geqslant 2$ consecutive sessions). Cocaine seeking (non-reinforced active lever responding) was then assessed in the COC-paired context. (b) Photomicrograph and schematics depicting cannula placement. The symbols denote the most ventral point of the injector cannula tracks for rats that received bilateral VEH ( $n=6$, white circles) or PP2 ( $n=8$, black circles) infusions into the $\mathrm{DH}$ following cocaine memory reactivation in Experiment la. Numbers indicate the distance from bregma (in mm), according to the rat brain atlas of Paxinos and Watson (1997). (c) Active and ( $c^{\prime}$ ) inactive lever responses during SA (mean \pm SEM per $2 \mathrm{~h}$ session, average of the last three training sessions), during the cocaine-memory reactivation session (MEM REACT; mean \pm SEM per 15 min session), in the extinction context (EXT; mean \pm SEM per $2 \mathrm{~h}$ session, the last session before the test in the COC-paired context), and in the COC-paired context (mean \pm SEM per $2 \mathrm{~h}$ session) for rats in Experiment Ia. (d) Active and (d') inactive lever responses in 20-min intervals during the test session in the COC-paired context. ${ }^{\dagger}$ Significant

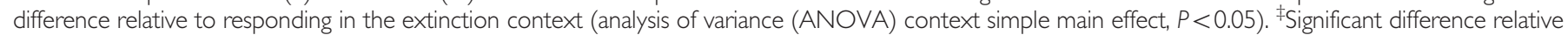
to VEH treatment (c, ANOVA treatment simple main effect, $P<0.05$; d, ANOVA treatment main effect, $P<0.05$ ).

amnesia). Furthermore, the extinction criterion results in uniform memory age at test because most rats reach the criterion in 2 days $(2.14 \pm 0.14$ days in the present study) and permits demonstration of statistically significant drug context-induced reinstatement of drug-seeking behavior at test by serving as reference. Twenty-four hours after the last extinction session, rats were placed into the cocaine-paired context for a 1-h test of drug contextinduced cocaine seeking, during which non-reinforced active lever presses were measured as an index of motivation for cocaine.

\section{Experiment 1b}

In Experiment 1b, we tested whether the effects of PP2 was dependent on explicit cocaine-memory reactivation, consistent with a genuine memory reconsolidation impairment
(Nader et al, 2000b). The experimental procedures and timeline (Figure 2a) followed those in Experiment 1a, except that the rats were exposed to an unpaired context (Context C; see Supplementary Table S1), which differed from Contexts A and $\mathrm{B}$, for $15 \mathrm{~min}$ before receiving bilateral microinfusions of VEH or PP2 into the DH. Exposure to the unpaired context was expected to provide a similar behavioral experience to that of the cocaine-memory reactivation session without explicit cocaine-memory reactivation.

\section{Experiment 2}

In Experiment 2, we evaluated the effects of cocaine-memory reactivation and PP2 treatment on the phosphorylation of SFK substrates: GluN2A (Taniguchi et al, 2009) and GluN2B NMDAR subunits (Nakazawa et $a l, 2001$ ) and GluA2 AMPAR subunits (Hayashi et al, 1999). We hypothesized 
a Self-Administration Extinction No memory reactivation

\begin{tabular}{|c|c|c|c|}
\hline $12345678910^{\circ} 1234567$ & $\begin{array}{c}\text { 15-min re-exposure } \\
\text { to unpaired ctx }\end{array}$ & $12^{\#}$ & $\begin{array}{c}\text { 1-h test } \\
\text { of cocaine seeking }\end{array}$ \\
PP2 or VEH \\
into the DH
\end{tabular}

b

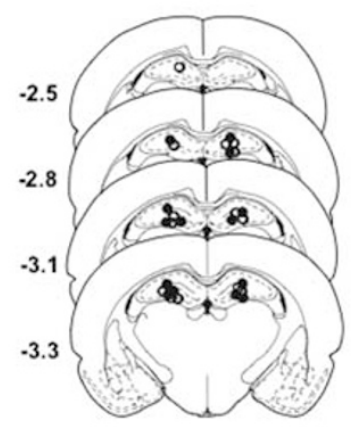

C

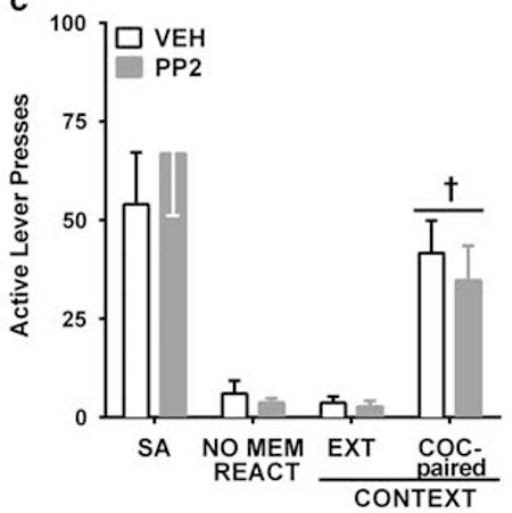

c

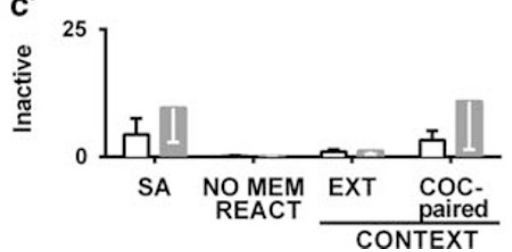

Figure 2 Inhibition of Src family of tyrosine kinases (SFKs) in the dorsal hippocampus (DH) in the absence of explicit cocaine-memory reactivation fails to alter subsequent drug context-induced cocaine seeking. (a) Schematic depicting the timeline for Experiment Ib. Experimental parameters were identical to those in Experiment la, except that rats in Experiment Ib were placed into an unpaired context (No memory reactivation) for 15 min before receiving bilateral microinfusions of PP2 (a nonselective SFK inhibitor; $62.5 \mathrm{ng}$ per $0.5 \mu \mathrm{l}$ per hemisphere) or vehicle (VEH) $(0.5 \mu \mathrm{l}$ per hemisphere) into the DH. (b) Schematics depicting cannula placement. The symbols denote the most ventral point of the injector cannula tracks for rats that received bilateral VEH ( $n=7$, white circles) or PP2 ( $n=7$, black circles) infusions into the DH following unpaired context exposure in Experiment Ib. Numbers indicate the distance from bregma (in mm), according to the rat brain atlas of Paxinos and Watson (1997). (c) Mean active lever responses during SA (mean \pm SEM per $2 \mathrm{~h}$ session, average of the last three training sessions), during unpaired context exposure (NO MEM REACT; mean \pm SEM per I 5 min session), in the extinction context (EXT; mean \pm SEM per $2 \mathrm{~h}$ session, the last session before the test in the COC-paired context), and in the COC-paired context (mean \pm SEM per $2 \mathrm{~h}$ session) for rats in Experiment Ib. $\left(c^{\prime}\right)$ Inactive lever responses in Experiment Ib. ${ }^{\dagger}$ Significant difference relative to responding in the extinction context (analysis of variance (ANOVA) context main effect, $P<0.05)$.

that cocaine-memory reconsolidation would increase GluN2A subunit phosphorylation and this would be attenuated by PP2. The experimental parameters and timeline in Experiment 2 followed those in Experiments 1a, except that rats were exposed to the cocaine-paired context or the home cage before intra-DH VEH or PP2 microinfusions (Figure 3a). The home cage condition provides an index of basal SFK substrate phosphorylation. Rats were killed $15 \mathrm{~min}$ or $1 \mathrm{~h}$ following intracranial microinfusions to capture a range of possible phosphorylation kinetics of SFK substrates (Hayashi and Huganir, 2004; Schumann et al, 2008, 2009; Xie et al, 2013) related to memory reconsolidation per se (Pedreira and Maldonado, 2003). Following rapid decapitation, brains were removed, flash frozen in isopentane, and stored at $-80^{\circ} \mathrm{C}$.

\section{Western Blotting}

Tissue punches of the DH or the SStr were taken using $19 \mathrm{Ga}$ neuropunches (Fine Science Tools) from $40 \mu \mathrm{m}$ tissue sections, collected to verify cannula placement. DH punches included the approximate volume of PP2 diffusion around the injector needle and contained parts of CA1, CA3, and the dentate gyrus. Tissue storage and preparation are described in Supplementary Methods. Thirty micrograms of protein from each sample were electrophoresed on a $7.5 \%$ Tris- $\mathrm{HCl}$ polyacrylamide gel and transferred to a polyvinylidene difluoride membrane for $1 \mathrm{~h}$ at $100 \mathrm{~V}$. Membranes were blocked in 5\% nonfat milk or Odyssey blocking buffer (LICOR Biotechnology, Lincoln, NE) for $1 \mathrm{~h}$ and incubated in polyclonal antibodies developed in rabbit against phosphorylated GluN2A (pTyr1325), GluN2B (pTyr1472), or GluA2 (pTyr876) (1:1000) each overnight $(16-20 \mathrm{~h})$ at $4{ }^{\circ} \mathrm{C}$. Membranes were then incubated in anti-rabbit horseradish peroxidase-conjugated $(1: 10000)$ or infrared secondary antibody (1:20000) for $1 \mathrm{~h}$, followed by development with enhanced chemiluminescence (Pierce Biotech, Rockford, IL) or digital infrared imaging systems (LI-COR). Membranes were incubated with stripping buffer $(62.5 \mathrm{mM}$ Tris- $\mathrm{HCl}$ at $\mathrm{pH} 6.7,2 \%$ SDS, $100 \mathrm{mM} \beta$-mercaptoethanol) and then reprobed with antibodies developed in rabbit against total GluN2A or GluN2B (1:1000 each), antibodies developed in 
a

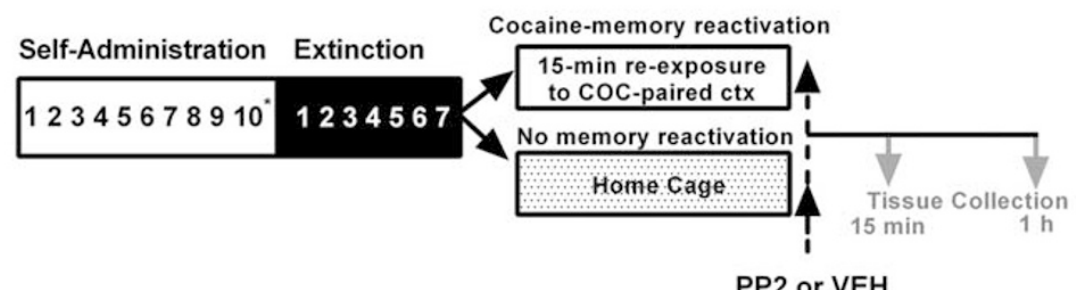

into the $\mathrm{DH}$

b
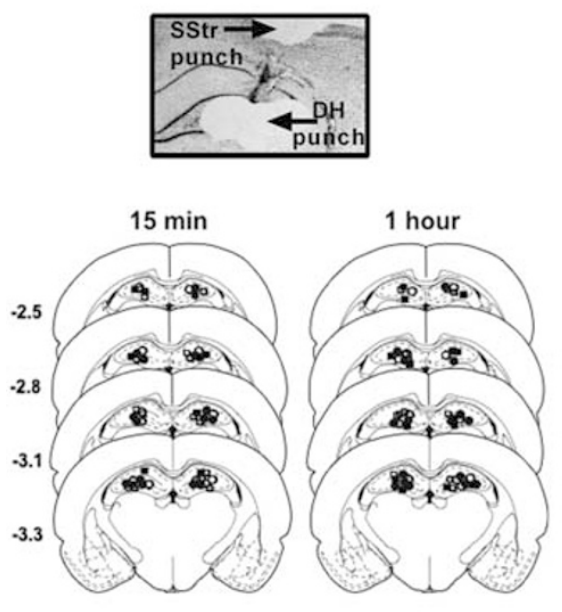

C

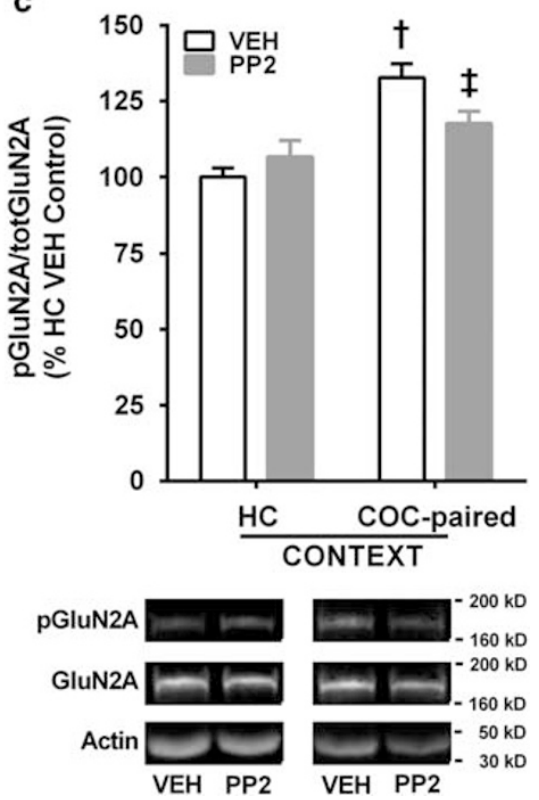

Figure 3 Effects of cocaine-memory reactivation and Src family of tyrosine kinase (SFK) inhibition in the dorsal hippocampus (DH) on GluN2A subunit phosphorylation. (a) Schematic depicting the timeline for Experiment 2. Experimental parameters were the same as those used in Experiment Ia, except that rats received bilateral microinfusions of PP2 (62.5 ng per $0.5 \mu$ per hemisphere) or vehicle $(V E H)$ into the DH after re-exposure to the cocaine-paired context (cocaine-memory reactivation) or the home cage (no memory reactivation). (b) Representative photomicrograph depicting the location of the tissue punches in the DH and SStr (trunk region of the somatosensory cortex) and schematics depicting cannula placement for rats that were killed 15 min after receiving cocaine-memory reactivation plus bilateral VEH ( 15 min: $n=4$, open circles) or PP2 ( 15 min: $n=8$, filled circles) infusions into the DH or following exposure to the home-cage plus bilateral VEH (I 5 min: $n=6$, open squares) or PP2 ( 5 min: $n=6$, filled squares) infusions. Numbers indicate the distance from bregma (in $\mathrm{mm}$ ), according to the rat brain atlas of Paxinos and Watson (1997). (c) Western blot analysis results show mean ( \pm SEM) GluN2A phosphorylation (phosphorylated per total protein levels normalized to actin, expressed as percentage of the comparator group (home cage $\mathrm{VEH}$ )) in the DH in rats killed $15 \mathrm{~min}$ after exposure to the cocaine-paired context (cocaine-memory reactivation) or home cage (no memory reactivation) plus VEH or PP2 treatment. ${ }^{\dagger}$ Significant difference relative to home cage (analysis of variance (ANOVA) context simple main effect, $P<0.05$ ). ${ }^{\ddagger}$ Significant difference relative to VEH treatment (ANOVA treatment simple main effect, $P<0.05$ ).

mouse against total GluA2 (1:1000), and later actin (loading control; 1:25 000). Manufacturer, catalog, and lot information for each antibody is provided in Supplementary Methods.

Protein levels were quantified by densitometry, using NIH Image J or Image Studio Version 4.0 software. To evaluate changes in protein phosphorylation, phosphorylated protein was normalized to total protein and to actin. Protein expression is discussed below in terms of normalized levels expressed as a percentage of the comparator group (ie, \% home cage VEH group).

\section{Experiment $3 a$}

In Experiment 3a, we examined whether antagonism of GluN2A subunit-containing NMDARs during cocainememory reconsolidation impairs subsequent drug contextinduced cocaine-seeking behavior. The experimental parameters and timeline (Figure 4a) followed those in Experiment 1a, except that rats received bilateral microinfusions of phosphate-buffered saline VEH or the GluN2A subunit-preferring NMDAR antagonist (Frizelle et al, 2006), PEAQX tetrasodium hydrate $(2.5 \mu \mathrm{g}$ per $0.5 \mu \mathrm{l}$ per hemisphere; Sigma-Aldrich, St Louis, MO), into the $\mathrm{DH}$ immediately after cocaine-memory reactivation. This PEAQX dose was selected because it attenuates cued fearmemory restabilization (Milton et al, 2013). A third group received bilateral microinfusions of PEAQX into the dorsally adjacent SStr following cocaine-memory reactivation to assess the anatomical specificity of PEAQX effects.

\section{Experiment 3b}

In Experiment 3b, we tested whether the effects of intra-DH PEAQX in Experiment 3a required explicit cocaine-memory 

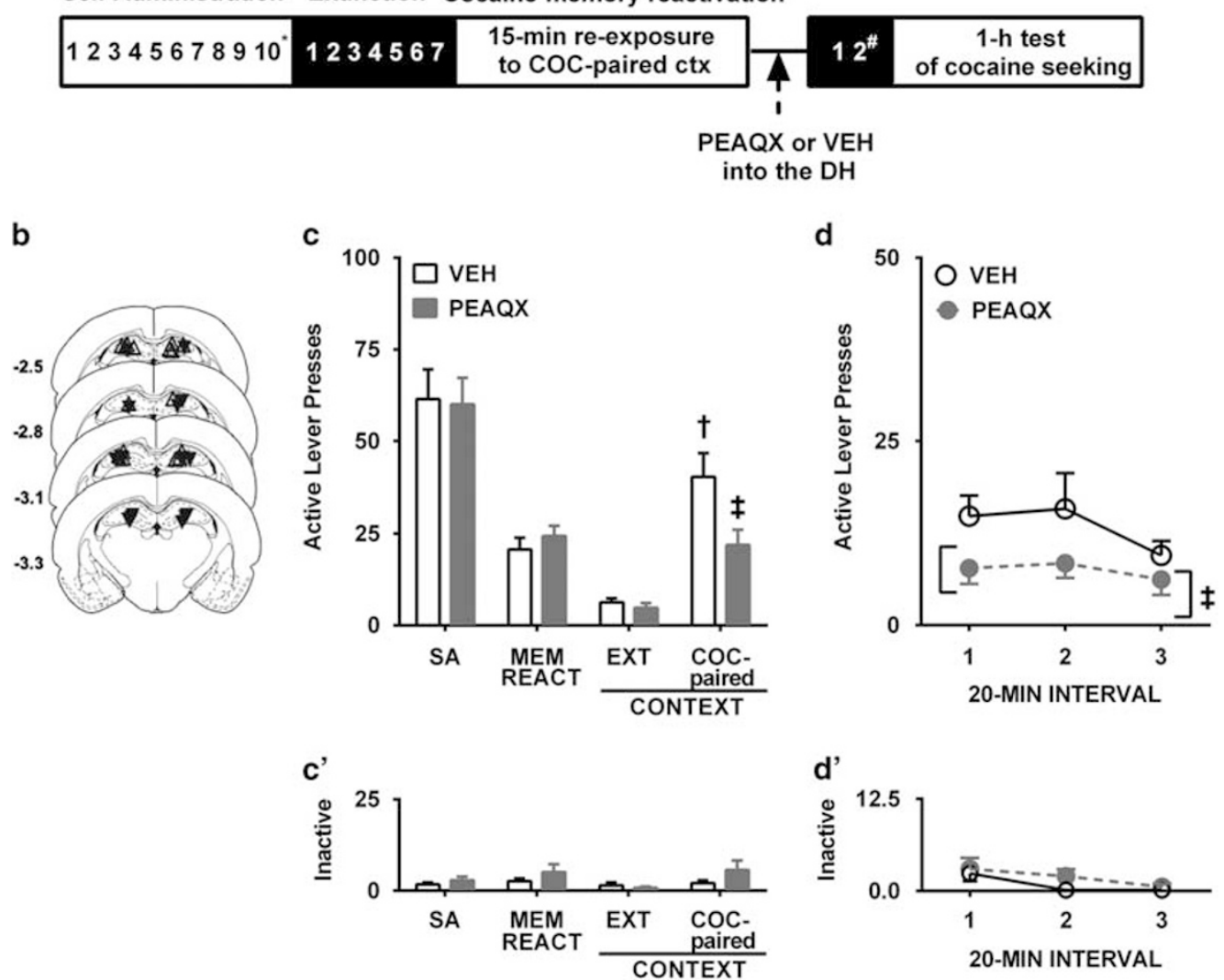

Figure 4 Antagonism of GluN2A-containing N-methyl-D-aspartate receptors (NMDARs) in the dorsal hippocampus (DH) following cocaine-memory reactivation impairs subsequent drug context-induced cocaine seeking. (a) Schematic depicting the timeline for Experiment 3a. Experimental parameters were identical to those in Experiment la, except that rats received bilateral microinfusions of PEAQX (a GluN2A-preferring NMDAR antagonist; $2.5 \mu \mathrm{g}$ per $0.5 \mu$ l per hemisphere), or phosphate-buffered saline vehicle (VEH; $0.5 \mu$ per hemisphere) into the DH after cocaine-memory reactivation. (b) Schematics depicting cannula placement. The symbols denote the most ventral point of the injector cannula tracks for rats that received bilateral vehicle $(V E H ; n=8)$ or PEAQX $(n=9$, black triangles) infusions into the DH following cocaine-memory reactivation in Experiment 3a. Numbers indicate the distance from bregma (in mm), according to the rat brain atlas of Paxinos and Watson (1997). (c) Active and ( $\left.c^{\prime}\right)$ inactive lever responses during SA (mean \pm SEM per $2 \mathrm{~h}$ session, average of the last three training sessions), during the cocaine-memory reactivation session (MEM REACT; mean \pm SEM per 15 min session), in the extinction context (EXT; mean \pm SEM per $2 \mathrm{~h}$ session, the last session before the test in the COC-paired context), and in the COC-paired context (mean \pm SEM per $2 \mathrm{~h}$ session ) for rats in Experiment $3 \mathrm{a}$. (d) Active and $\left(d^{\prime}\right)$ inactive lever responses in 20-min intervals during the test session in the cocaine-paired context. 'Significant difference relative to responding in the extinction context (analysis of variance (ANOVA) context main effect, $P<0.05$ ). ${ }^{\circledR}$ Significant difference relative to VEH treatment (c, ANOVA treatment simple main effect, $P<0.05$; $d$, ANOVA treatment main effect, $P<0.05)$.

reactivation. The experimental parameters and timeline (Figure 5a) followed those in Experiment 3a, except that rats received bilateral microinfusions of VEH or PEAQX into the DH after unpaired context exposure.

\section{Histology}

Rats in Experiments 1 and 3 were overdosed with ketamine hydrochloride and xylazine $(75$ and $5 \mathrm{mg} / \mathrm{kg}$, intravenously or 150 and $10 \mathrm{mg} / \mathrm{kg}$, intraperitoneally, respectively, depending on catheter patency). Brains were removed, postfixed with $10 \%$ formaldehyde solution, and sectioned at $75 \mu \mathrm{m}$. Sections were stained using cresyl violet (Kodak, Rochester, NY). Cannula tracks were mapped onto schematics from the rat brain atlas (Paxinos and Watson, 1997).

\section{Data Analysis}

Data were analyzed using analyses of variance (ANOVAs) with Tukey's post hoc tests or $t$-tests, where appropriate (see Supplementary Methods for details). Alpha was set at 0.05 .

\section{RESULTS}

\section{Histology}

Cannula tracks terminated within the $\mathrm{DH}$ proper, which included portions of the CA1, CA3, and dentate gyrus subregions (Figures 1, 2, 3, 4 and 5b). High-power microscopy did not reveal tissue damage extensive cell loss or gliosis at injection sites. Data from rats with incorrect cannula placements were excluded from statistical analysis.

\section{Behavioral History}

There were no pre-existing differences between the experimental groups in Experiments 1, 2, or 3 in cocaine intake or in active or inactive lever responses during selfadministration or extinction training or during the memory reactivation session. Furthermore, these groups did not differ in the number of days required to reach the extinction criterion aftertreatment (all Fs or ts $\leqslant 1.76, P_{\mathrm{s}} \geqslant 0.10$ ). 
a
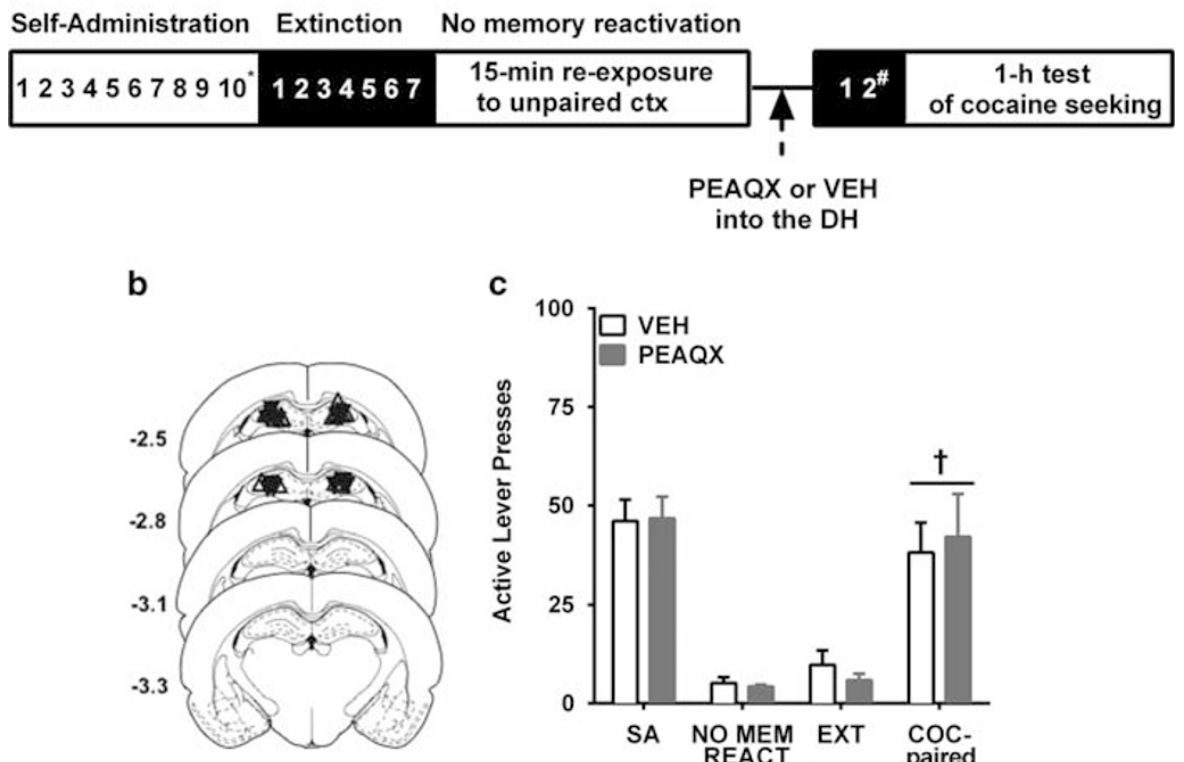

C
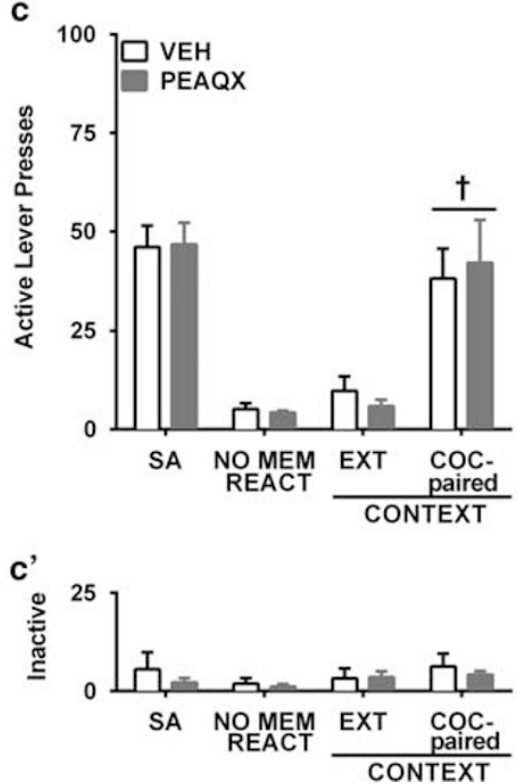

Figure 5 Antagonism of GluN2A-containing N-methyl-D-aspartate receptors (NMDARs) in the dorsal hippocampus (DH) without cocaine-memory reactivation has no effect on subsequent drug context-induced cocaine seeking. (a) Schematic depicting the timeline for Experiment 3b. Experimental parameters were identical to those in Experiment 3a, except that rats in Experiment $3 b$ received exposure to the unpaired context (no memory reactivation) for 15 min before receiving bilateral microinfusions of PEAQX (a GluN2A subunit-preferring NMDAR antagonist; $2.5 \mu \mathrm{g}$ per $0.5 \mu$ l per hemisphere) or vehicle (VEH; $0.5 \mu \mathrm{l}$ per hemisphere) into the DH. (b) Schematics depicting cannula placement. The symbols denote the most ventral point of the injector cannula tracks for rats that received bilateral VEH ( $n=8$, white circles) or PEAQX $(n=8$, black circles) infusions into the DH following unpaired context exposure in Experiment 3b. Numbers indicate the distance from bregma (in mm), according to the rat brain atlas of Paxinos and Watson (1997). (c) Active lever responses during SA (mean \pm SEM per $2 \mathrm{~h}$ session, average of the last three training sessions), during unpaired context exposure (NO MEM REACT; mean \pm SEM per 15 min session), in the extinction context (EXT; mean \pm SEM per $2 \mathrm{~h}$ session, the last session before the test in the COC-paired context), and in the COCpaired context (mean \pm SEM per $2 \mathrm{~h}$ session) for rats in Experiment $3 b$. $\left(c^{\prime}\right)$ Inactive lever responses in Experiment $3 b$. ${ }^{\dagger}$ Significant difference relative to responding in the extinction context (analysis of variance (ANOVA) context main effect, $P<0.05$ ).

\section{SFK Inhibition in the DH Following Cocaine Context Re-Exposure or Unpaired Context Exposure Differentially Alters Subsequent Drug Context-Induced Cocaine Seeking}

Intra-DH microinfusions of PP2 administered following re-exposure to the previously cocaine-paired context- (ie, cocaine-memory reactivation) attenuated subsequent cocaineseeking behavior in a context-dependent manner (Figure 1c; ANOVA context $\times$ treatment interaction, $\mathrm{F}_{(1,12)}=5.88$, $P=0.03$; context main effect, $\mathrm{F}_{(1,12)}=25.13, P<0.01$; treatment main effect, $\left.\mathrm{F}_{(1,12)}=4.89, \quad P=0.05\right)$. Exposure to the cocaine-paired context increased active lever responding in the group that had received VEH into the $\mathrm{DH}$ following cocaine-memory reactivation, relative to responding in the extinction context on the preceding day (Tukey's test, $P<0.05)$. The PP2-treated group exhibited less active lever responding in the cocaine-paired, but not in the extinction, context, relative to the VEH-treated group (Tukey's test, $P<0.05)$. Active lever responding in the PP2-treated group did not differ between the extinction and cocaine-paired contexts. Time-course analysis of active lever responding during the test session in the cocaine-paired context confirmed that the PP2treated group responded less on the active lever compared with the VEH-treated group, independent of time (Figure 1d; ANOVA treatment main effect only, $\mathrm{F}_{(1,12)}=5.48, P=0.04$; all time main and interaction effects, $\mathrm{F}_{(2,24)}=0.03-3.04$, $P=0.07-0.95)$.

Intra-DH microinfusions of PP2 administered following exposure to the unpaired context (ie, absence of explicit cocaine-memory reactivation) failed to alter subsequent cocaine-seeking behavior (Figure 2c). At test, exposure to the cocaine-paired context significantly increased active lever responding in both the VEH- and PP2-treated groups, relative to responding in the extinction context (ANOVA context main effect only, $\left.\mathrm{F}_{(1,12)}=33.47, P<0.01\right)$. Moreover, there was no difference between the groups in active lever responding in either context (all treatment main and interaction effects, $\left.\mathrm{F}_{(1,12)}=0.25-0.42, P=0.53-0.63\right)$. 
Effects of Cocaine-Memory Reactivation and SFK Inhibition in the DH on GluN2A, GluN2B, and GluA2 Subunit Phosphorylation

Quantitative western blot analyses indicated that total GluN2A subunit levels did not differ across the groups (all Fs $<3.53, P_{s}>0.08$ ) but GluN2A subunit phosphorylation in the $\mathrm{DH}$ was dependent on context exposure and treatment (Figure 3c; ANOVA context $\times$ treatment interaction, $\mathrm{F}_{(1,20)}=5.54, P=0.03$; context main effect, $\mathrm{F}_{(1,20)}=22.99$, $P=0.0001)$. Re-exposure to the cocaine-paired context increased GluN2A subunit phosphorylation, relative to home cage exposure, 15 min after treatment (Tukey's test, $P<0.05$ ). Moreover, intra-DH PP2 treatment reduced GluN2A subunit phosphorylation following exposure to the cocaine-paired context (Tukey's test, $P<0.05$ ), but not the home cage, relative to VEH. However, intra-DH PP2 administered following exposure to the cocaine-paired context failed to alter GluN2A subunit phosphorylation in the dorsally adjacent SStr $15 \mathrm{~min}$ after treatment (Supplementary Table S2; $t(10)=1.17, P=0.27)$.

Neither cocaine context re-exposure nor PP2 treatment altered GluN2A subunit phosphorylation $1 \mathrm{~h}$ after treatment (Supplementary Table S2; Fs $<3.12, P s>0.09$ ). Similarly, neither cocaine context re-exposure nor PP2 treatment altered GluN2B or GluA2 subunit phosphorylation at either time point (Supplementary Table S2; all Fs $<2.36, P s>0.14$ ).

GluN2A Subunit-Containing NMDAR Antagonism in the DH Following Cocaine Context Re-Exposure or Unpaired Context Exposure Differentially Alters Subsequent Drug Context-Induced Cocaine Seeking

Intra-DH PEAQX administration following cocaine-memory reactivation attenuated subsequent cocaine-seeking behavior in a context-dependent manner (Figure 4c; ANOVA context $\times$ treatment interaction, $\mathrm{F}_{(1,15)}=5.13, P=0.04$; context main effect, $F_{(1,15)}=47.00, P<0.01$; treatment main effect, $\left.\mathrm{F}_{(1,15)}=6.17, P=0.03\right)$. Exposure to the cocaine-paired context significantly increased active lever responding in the group that had received VEH into the $\mathrm{DH}$ following cocainememory reactivation, relative to responding in the extinction context on the preceding day (Tukey's test, $P<0.05$ ). The PEAQX-treated group exhibited less active lever responding in the cocaine-paired, but not in the extinction context, relative to the VEH-treated group (Tukey's test, $P<0.05$ ). Active lever responding in the PEAQX-treated group did not significantly differ between the two contexts. Time-course analysis of active lever responding during the test session in the cocaine-paired context revealed that the PEAQX-treated group exhibited fewer active lever responses than the VEHtreated group, independent of time (Figure 4d; ANOVA treatment main effect only, $\mathrm{F}_{(1,15)}=5.73, P=0.03$; all time main and interaction effects, $F_{(2,30)}=0.40-1.56, P=0.23-$ 0.68). Furthermore, a third group that had received PEAQX into the dorsally adjacent SStr $(n=6)$ exhibited more active lever responses in the cocaine-paired context (mean $\pm \mathrm{SEM}=$ $37.33 \pm 9.43$; data not shown) compared with that in the extinction context $\left(t_{(5)}=3.07, P=0.03\right)$, similar to the VEHtreated group.

Intra-DH PEAQX administration following exposure to the unpaired context failed to alter subsequent cocaine-seeking behavior relative to VEH (Figure 5c). At test, exposure to the cocaine-paired context significantly increased active lever responding in both VEH- and PEAQX-treated groups, relative to responding in the extinction context (ANOVA context main effect only, $\left.F_{(1,14)}=24.22, P<0.01\right)$. Moreover, the groups did not differ in active lever responding in either context (all treatment main and interaction effects, $\left.\mathrm{F}_{(1,14)}=0.00-0.36, P=0.56-0.99\right)$.

\section{Inactive Lever Responding}

Inactive lever responding was low in all experiments. The groups did not differ as a function of treatment, testing context, or time (all Fs $<3.35, P s>0.05$; Figures 1 and $2 c^{\prime}$, Figures 4 and $5 c^{\prime}$, and Figures $1 d^{\prime}$ and $\left.4 d^{\prime}\right)$.

\section{DISCUSSION}

In the present study, we demonstrate that activation of hippocampal SFKs and stimulation of GluN2A-containing NMDARs are required for cocaine-memory reconsolidation and drug context-induced instrumental cocaine-seeking behavior. In Experiment 1a, administration of the SFK inhibitor, PP2, into the $\mathrm{DH}$ of rats following 15-min reexposure to the cocaine-associated context-attenuated drug context-induced cocaine-seeking behavior $\sim 72 \mathrm{~h}$ later, relative to VEH (Figure 1c). Postsession PP2 administration was likely time-locked to the onset of memory reconsolidation (Fuchs et al, 2009). Furthermore, while lever pressing was not reinforced during memory reactivation, it is unlikely that postsession PP2 administration enhanced extinction memory consolidation. The 15 -min memory reactivation session did not result in observable extinction learning; thus, it is improbable that the extinction memory trace was the dominant memory trace at the onset of memory reconsolidation (Eisenberg et al, 2003). Moreover, extant literature indicates that postsession intra-DH PP2 administration impairs, rather than enhances, extinction memory consolidation in other experimental procedures (Bevilaqua et al, 2005). A similar effect would have been indicated by increase, rather than the observed decrease (Figure 1c), in active lever pressing at test in our model. Similarly, PP2 effects cannot be attributed to protracted hypoactivity or general amnesia, as PP2 administration following unpaired context exposure (ie, no memory reactivation) did not attenuate subsequent drug context-induced cocaine-seeking behavior, relative to VEH (Figure 2c). PP2 also failed to alter active lever responding in the extinction context (Figure 1c) and inactive lever responding in either context, relative to VEH (Figure 1 $c^{\prime}$ ), albeit these measures are subject to floor effects. Finally, diffusion of PP2 away from the DH to other brain regions was negligible. Consistent with this, intra-DH PP2 treatment did not alter GluN2A subunit phosphorylation in the dorsally adjacent SStr, which falls in the most probable path of drug diffusion along the cannula track (Supplementary Table S2). Overall, these findings suggest that PP2 in the DH selectively disrupted the reconsolidation of cocaine memories.

GluN2A NMDAR subunit activation was also necessary for cocaine-memory reconsolidation (Figure 4c). In Experiment $3 \mathrm{a}$, administration of the GluN2A-preferring 
antagonist, PEAQX, into the $\mathrm{DH}$ of rats following cocainememory reactivation attenuated subsequent drug context-induced cocaine-seeking behavior, relative to VEH. Recapitulating the effects of PP2, PEAQX effects were dependent on explicit cocaine-memory reactivation, in that PEAQX treatment administered after unpaired context exposure was without effect at test (Figure 5c). PEAQX effects were also anatomically selective, such that PEAQX treatment administered into the SStr did not attenuate cocaine seeking at test (data not shown). These findings extend research demonstrating the involvement of GluN2Acontaining NMDARs in the BLA in auditory fear-memory reconsolidation (Milton et al, 2013).

SFKs tightly regulate GluN2A NMDAR subunit activation (Taniguchi et al, 2009). Thus, we examined the phosphorylation state of GluN2A subunits, as well as alternate SFK substrates-GluN2B NMDAR and GluA2 AMPAR subunits-following cocaine-memory reactivation or no memory reactivation and intra-DH PP2 or VEH treatment. Cocaine-memory reactivation increased GluN2A subunit phosphorylation compared with no memory reactivation at $15 \mathrm{~min}$, but not at $1 \mathrm{~h}$ (Supplementary Table S2), postmemory reactivation (Figure $3 \mathrm{c}$ ). Transient GluN2A activation is consistent with the rapid phosphorylation/ dephosphorylation kinetics of GluN2A NMDAR subunits (Vincini et al, 1998). Src-mediated phosphorylation of GluN2A subunits promotes NMDAR surface expression (Taniguchi et al, 2009), potentiates NMDAR currents in the striatum in vivo (Taniguchi et al, 2009), and enhances LTP at CA1 synapses in vitro (Yang et al, 2012). Among others, LTP-like processes promote memory reconsolidation (Nader et al, 2000a; Alberini, 2006; Abraham and Williams, 2008). Thus, the increase in GluN2A phosphorylation following cocaine-context exposure likely reflects obligatory GluN2A regulation during memory reconsolidation. In support of this hypothesis, intra-DH PP2 treatment administered following exposure to the cocaine-paired context, but not the home cage, attenuated both the transient increase in GluN2A subunit activation in the $\mathrm{DH}$ (Figure $3 \mathrm{c}$ ) and subsequent cocaine-seeking behavior in a separate cohort of rats (Figure $1 \mathrm{c}$ and $\mathrm{d}$ ). Furthermore, the results of the PEAQX experiment (Figure 4c) indicated that a minimum current through a particular sub-population of GluN2A-containing NMDA receptors is required for successful cocaine-memory reconsolidation.

PP2 is a global SFK inhibitor of Src, Fyn, Lyn, Yes, and Lck (Hanke et al, 1996; Bain et al, 2003). Specific SFKs differently control the activity GluN2A, GluN2B, and GluA2 subunits, permitting bidirectional control over memory strength through synaptic depotentiation and potentiation (for a review, see Ohinishi et al, 2011). Our findings implicate GluN2A NMDAR subunits in cocaine-memory restabilization, and existing literature indicates that the activation of these subunits is selectively mediated by Src per se in the hippocampus (Yang et al, 2012; Trepanier et al, 2013). However, other SFKs may promote memory destabilization by Lyn-dependent GluA2 AMPAR subunit phosphorylation (Hayashi et al, 1999; Hayashi and Huganir, 2004; Fox et al, 2007) or Fyn-dependent of GluN2B NMDAR subunit phosphorylation (Yaka et al, 2002; Lavezzari et al, 2003; Prybylowski et al, 2005; Zhang et al, 2008), which promote LTD-like processes (Shi et al, 2001; Liu et al, 2004; Kim et al,
2005; Yang et al, 2012). Therefore, future studies will be essential to elucidate the contribution of specific SFKs to discrete memory processes (eg, destabilization and reconsolidation) involved in the prolonged maintenance of salient and intrusive drug-related memories.

It is noteworthy that neither cocaine-memory reactivation nor PP2 treatment altered GluN2B or GluA2 subunit activation in the DH (Supplementary Table S2). Fyndependent phosphorylation of GluN2B-containing NMDARs, in particular, has been shown to support ethanol-seeking behaviors (Wang et al, 2007, 2010) and the context-induced reinstatement of cocaine-seeking behavior (Xie et al, 2013). The apparent lack of involvement of GluN2B subunits in the present study highlights distinct mechanisms governing the expression of relapse-like behaviors and the maintenance of long-term memories subserving this phenomenon.

In summary, the present findings point to SFKs and GluN2A-containing NMDARs as novel intracellular mechanisms through which the DH may critically support drug-memory reconsolidation (Ramirez et al, 2009), perhaps by supporting protein synthesis-dependent cocaine-memory restabilization in other brain regions, such as the BLA (Fuchs et al, 2009; Wells et al, 2011), or by promoting the establishment of retrieval links to recently restabilized cocaine memories (Wells et al, 2011). This may require direct phosphorylation of GluN2A subunits by Src (Taniguchi et al, 2009), although future studies will be necessary to show that this interaction is requisite. Nevertheless, the present findings begin to clarify the complex role of the $\mathrm{DH}$ in cocaine-memory reconsolidation and may inform the development of treatments intended to weaken drug-related memories.

\section{FUNDING AND DISCLOSURE}

This research was funded by NIDA R01 DA025646 (RAF) and NIDA F31 DA034391 (AMW). The authors declare no conflict of interest.

\section{ACKNOWLEDGMENTS}

We thank Dr Kelley Harmon for excellent technical assistance.

\section{REFERENCES}

Abraham WC, Williams JM (2008). LTP maintenance and its protein synthesis-dependence. Neurobiol Learn Mem 89: 260-268. Alberini CM (2006). The role of protein synthesis during the labile phases of memory: revisiting the skepticism. Neurobiol Learn Mem 89: 234-246.

Bain J, McLauchlan H, Elliott M, Cohen P (2003). The specificities of protein kinase inhibitors: an update. Biochem J 371: 199-203. Bevilaqua LRM, da Silva WN, Medina JH, Izquierdo I, Cammarota M (2005). Extinction and reacquisition of a fearmotivated memory require activity of the Src family of tyrosine kinases in the CA1 region of the hippocampus. Pharmacol, Biochem Behav 81: 139-145.

Brown TE, Lee BR, Sorg BA (2008). The NMDA antagonist MK-801 disrupts reconsolidation of a cocaine-associated memory for conditioned place preference but not for self-administration in rats. Learn Mem 15: 857-865. 
Childress A, Ehrman R, McLellan AT, O’Brien C (1988). Conditioned craving and arousal in cocaine addiction: a preliminary report. NIDA Res Monogr 81: 74-80.

Eisenberg M, Kobilo T, Berman DE, Dudai Y (2003). Stability of retrieved memory: inverse correlation with trace dominance. Science 301: 1102-1104.

Fox CJ, Russel K, Titterness AK, Wang YT, Christie BR (2007). Tyrosine phosphorylation of the GluR2 subunit is required for long-term depression of synaptic efficacy in young animals in vivo. Hippocampus 17: 600-605.

Frizelle PA, Chen PE, Wyllie DJ (2006). Equilibrium constants for (R)-[(S)-1-(4-bromo-phenyl)-ethylamino]-(2,3-dioxo-1,2,3,4tetrahydroquino xalin-5-yl)-methyl]-phosphonic acid (NVPAAM077) acting at recombinant NR1/NR2A and NR1/NR2B $\mathrm{N}$-methyl-D-aspartate receptors: implications for studies of synaptic transmission. Mol Pharmacol 70: 1022-1032.

Fuchs RA, Bell GH, Ramirez DR, Eaddy JL, Su ZI (2009). Basolateral amygdala involvement in memory reconsolidation processes that facilitate drug context-induced cocaine seeking. Eur J Neurosci 30: 889-900.

Gawin FH, Kleber HD (1986). Abstinence symptomatology and psychiatric diagnosis in cocaine abusers. Arch Gen Psychiatry 43: 107-113.

Hanke JH, Gardner JP, Dow RL, Changelian PS, Brissette WH, Weringer EJ et al (1996). Discovery of a novel, potent, and Src family-selective tyrosine kinase inhibitor. Study of Lck- and FynT-dependent T cell activation. J Biol Chem 271: 695-701.

Hayashi T, Huganir R (2004). Tyrosine phosphorylation and regulation of the AMPA receptor by Src family tyrosine kinases. J Neurosci 24: 6152-6160.

Hayashi T, Umemori H, Mishina M, Yamamoto T (1999). The AMPA receptor interacts with and signals through the protein tyrosine kinase Lyn. Nature 397: 72-76.

Inda MC, Muravieva EV, Alberini CM (2011). Memory retrieval and the passage of time: from reconsolidation and strengthening to extinction. J Neurosci 31: 1635-1643.

Kelley JB, Anderson KL, Itzhak Y (2007). Long-term memory of cocaine-associated contex: disruption and reinstatement. NeuroReport 18: 777-780.

Kim MJ, Dunah AW, Wang YT, Sheng M (2005). Differential roles of NR2A- and NR2B-containing NMDA receptors in Ras-ERK signaling and AMPA receptor trafficking. Neuron 46: 745-760.

Lavezzari G, McCallum J, Lee R, Roche KW (2003). Differential binding of the AP-2 adaptor complex and PSD-95 to the C-terminus of the NMDA receptor subunit NR2B regulates surface expression. Neuropharmacology 45: 729-737.

Liu L, Wong TP, Pozza MF, Lingenhoehi K, Wang Y, Sheng M et al (2004). Role of NMDA receptor subtypes in governing the direction of hippocampal synaptic plasticity. Science 304: 1021-1024.

Milton AL, Lee JL, Butler VJ, Gardner R, Everitt BJ (2008a). Intra-amygdala and systemic antagonism of NMDA receptors prevents the reconsolidation of drug-associated memory and impairs subsequently both novel and previously acquired drugseeking behaviors. J Neurosci 28: 8230-8237.

Milton AL, Merlo E, Ratano P, Gregory BL, Dumbreck JK, Everitt BJ (2013). Double dissociation of the requirement for GluN2Band GluN2A-containing NMDA receptors in the destabilization and restabilization of a reconsolidating memory. J Neurosci 33: $1109-1115$.

Nader K, Schafe GE, LeDoux JE (2000a). Fear memories require protein synthesis in the amygdala for reconsolidation after retrieval. Nature 406: 722-726.

Nader K, Schafe GE, LeDoux JE (2000b). The labile nature of consolidation theory. Nat Rev Neurosci 1: 216-219.

Nakazawa T, Komai S, Tezuka T, Hisatsune C, Umemori H, Semba K et al (2001). Characterization of Fyn-mediated tyrosine phosphorylation sites on GluR epsilon 2 (NR2B) subunit of the $N$ methyl-D-aspartate receptor. J Biol Chem 276: 693-699.

Ohinishi H, Murata Y, Okazawa H, Matozaki T (2011). Src family kinases: modulators of neurotransmitter receptor function and behavior. Trends Neurosci 34: 629-637.

Paxinos G, Watson C (1997). The Rat Brain in Stereotaxic Coordinates, Compact 3rd edn. Academic Press: San Diego, CA.

Pedreira ME, Maldonado H (2003). Protein synthesis subserves reconsolidation or extinction depending on reminder duration. Neuron 38: 863-869.

Prybylowski K, Sans N, Kan L, Vicini S, Wenthold RJ (2005). The synaptic localization of NR2b-containing NMDA receptors is controlled by interactions with PDZ proteins and AP-2. Neuron 47: 845-857.

Purcell AL, Carew TJ (2003). Tyrosine kinases, synaptic plasticity and memory: insights from vertebrates and invertebrates. Trends Neurosci 26: 625-630.

Ramirez DR, Bell GH, Lasseter HC, Xie X, Traina SA, Fuchs RA (2009). Dorsal hippocampal regulation of memory reconsolidation processes that facilitate drug context-induced cocaineseeking behavior in rats. Eur J Neurosci 30: 901-912.

Sadler R, Herzig V, Schmidt WJ (2007). Repeated treatment with the NMDA antagonist MK-801 disrupts reconsolidation of a memory for amphetamine-conditioned place preference. Behav Pharmacol 18: 669-703.

Salter MW, Kalia LV (2004). SRC kinases: a hub for NMDA receptor regulation. Nat Rev Neurosci 5: 317-328.

Schumann J, Alexandrovich GA, Biegon A, Yaka R (2008). Inhibition of NR2b phosphorylation restores alteration in NMDA receptor expression and improves functional recovery following traumatic brain injury in mice. J Neurotrauma 25: 945-957.

Schumann J, Michaeli A, Yaka R (2009). Src-protein tyrosine kinases are required for cocaine-induced increase in the expression and function of NMDA receptors in the ventral tegmental area. J Neurochem 108: 697-706.

Shi S, Hayashi Y, Esteban JA, Malinow R (2001). Subunit-specific rules governing AMPA receptor trafficking to synapses in hippocampal pyramidal neurons. Cell 105: 331-343.

Sorg BA (2012). Reconsolidation of drug memories. Neurosci Biobehav Rev 36: 1400-1417.

Stramiello M, Wagner JJ (2008). D1/5 receptor-mediated enhancement of LTP requires PKA, Src family kinases, and NR2Bcontaining NMDARs. Neuropharmacology 55: 871-877.

Suzuki A, Josselyn SA, Frankland PW, Masushige S, Silva AJ, Kida S (2004). Memory reconsolidation and extinction have distinct temporal and biochemical signatures. J Neurosci 24: 4787-4795.

Taniguchi S, Nakazawa T, Tanimura A, Kiyama Y, Tezuka T, Watabe AM et al (2009). Involvement of NMDAR2a tyrosine phosphorylation in depression-related behavior. EMBO $J \mathbf{2 8}$ : 3717-3729.

Trepanier C, Lei G, Xie YF, MacDonald JF (2013). Group II metabotropic glutamate receptors modify $N$-methyl-D-aspartate receptors via Src kinase. Sci Rep 3: 926.

Tronson NC, Taylor JR (2013). Addiction: a drug-induced disorder of memory reconsolidation. Curr Opin Neurobiol 23: 573-580.

Vincini S, Wang JF, Li JH, Zhu WJ, Wang YH, Luo JH et al (1998). Functional and pharmacological differences between recombinant $N$-methyl-D-aspartate receptors. J Neurophysiol 79: 555-566.

Wang J, Carnicella S, Phamluong K, Jeanblanc J, Ronesi JA, Chaudhri N, Janak PH, Lovinger DM, Ron D (2007). Ethanol induces long-term facilitation of NR2B-NMDA receptor activity in the dorsal striatum: implications for alcohol drinking behavior. J Neurosci 27: 3593-3602.

Wang J, Lanfranco MF, Gibb SL, Yowell QV, Carnicella S, Ron D (2010). Long-lasting adaptations of the NR2B-containing NMDA 
receptors in the dorsomedial striatum play a crucial role in alcohol consumption and relapse. J Neurosci 30: 10187-10198.

Wells AM, Lasseter HC, Xie X, Cowhey KE, Reittinger AM, Fuchs RA (2011). Interaction between the basolateral amygdala and dorsal hippocampus is critical for cocaine memory reconsolidation and subsequent drug context-induced cocaineseeking behavior in rats. Learn Mem 18: 693-702.

Xie X, Arguello AA, Wells AM, Reittinger AM, Fuchs RA (2013). Role of a hippocampal src-family kinase-mediated glutamatergic mechanism in drug context-induced cocaine seeking. Neuropsychopharmacology 38: 2657-2665.
Yaka R, Thornton C, Vagts AJ, Phamluong K, Bonci A, Ron D (2002). NMDA receptor function is regulated by the inhibitory scaffolding protein, RACK1. Proc Natl Acad Sci USA 99: 5710-5715.

Yang K, Trepanier C, Sidhu B, Xie YF, Li H, Lei G et al (2012). Metaplasticity gated through differential regulation of GluN2A versus GluN2B receptors by Src family kinases. EMBO J 31: 805-816.

Zhang S, Edelmann L, Liu J, Crandall JE, Morabito MA (2008). Cdk5 regulates the phosphorylation of tyrosine $1472 \mathrm{NR} 2 \mathrm{~B}$ and the surface expression of NMDA receptors. J Neurosci 28: 415-424.

Supplementary Information accompanies the paper on the Neuropsychopharmacology website (http://www.nature.com/npp) 